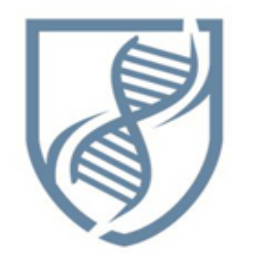

Journal of Bioscience and Applied Research



WWW.JBAAR.ORG

\title{
A new approach in bacteriological and chemical treatment of surface water for drinking purpose
}

\author{
Hisham.M ${ }^{1}$; Shaaban M.T. ${ }^{2}$; Sara. A ${ }^{3}$; Fakhrany.M ${ }^{4}$ and Hazaa.M.M. ${ }^{1}$ \\ 1-Botan y Dept. Fac. of Sci. Benha Univ., 2,- Botany Dept. Fac. of Sci. Menoufia Univ, 3- H.C of water and waste water, \\ 4- Geology Dept. Fac. of Sci. Benha Univ., Egypt .
}

Corresponding author e.mail.Dr.mohamedtawfiek@yahoo.com

\begin{abstract}
The surface water contain some dissolved contaminants such as iron and manganese. It is unsuitable for drinking water without appropriate treatment. Under standard and steady-state conditions, the bioreactor was very effective when $2 \%$ of the nano polymer composite granules (w/v)borne bioagent bacteria as a substrate for the biofilm formation. The mixture was aerated for $24 \mathrm{hs}$. The treated water have been uptake for determination the concentration of cations and microbiological analysis. Each 1L of surface water sample with continuous aeration and left for $24 \mathrm{~h}$ and as a result many physical, chemical and bacteriological changes occurred .For the surface water temperature decreased by $2.2^{\circ} \mathrm{C}$ and $2.0^{\circ} \mathrm{C}$ related to raw water and chlorinated water, $\mathrm{pH}$ increased by 0.2 and $0.4 \mathrm{ppm}$ related to raw water and chlorinated water. TDS decrease from (276 to $220 \mathrm{ppm}$ ) while in chemical treatment increase to (285 ppm) also conductivity decrease from(434 to 425$)$ $\mathrm{US} / \mathrm{CM}$ but increase to(442 US/CM) in the chlorinated treatment, while turbidity increase from (9.8 to 10.3) NTU. The elimination of the heavy metals was remarkable in this study as in rate of Fe that decrease from (0.39 to 0.21)ppm and for $\mathrm{Mn}$ it remains constant .Total alkalinity decrease from(142 to 130) ppm while in chemical treatment was 120 ppm. calcium hardness decrease from (78 to 54) ppm and as a result the calcium decrease from (31.2 to 21.6)ppm and in chemical one it has the same value of the raw water sample .Magnesium hardness increase from (100 to 108 )ppm \&for the magnesium ion increase from (10.56 to
\end{abstract}

16.8) $\mathrm{ppm}$. The chlorides decrease to $20 \mathrm{ppm}$ in the bio treated water sample but it was normal to increase to 31 ppm in the chemical treated sample. Sulfates content decrease from (15.5 to 10.7)ppm but in chemical treatment it increase to $17 \mathrm{ppm}$. Phosphate content increase from $(<0.01$ to 0.02$) \mathrm{ppm}$ while the nitrate content decrease from $(0.86$ to 0.5$) \mathrm{ppm}$ it also decrease in the chemical treatment to $0.48 \mathrm{ppm}$ and ammonia content decrease from (0.06 to0.01) ppm and in chemical treatment reach to $<0.01$ ppm a. Drinking water sources are contaminated with coliforms and pathogenic bacteria. The bacteriological results shows that all the bio treated sample had no fecal Coliform growth $(<1) \mathrm{C} / 100 \mathrm{ml}$ but give positive results with the total Coliform with confluent colonies whereas in the chemical treatment both the total \&fecal Coliform tests were negative.

Key words. Drinking water, Raw Surface water, Total coliform, Fecal Coliform

\section{Introduction}

Water is essential to sustain life, and availability of safe drinking water is very important. To ensure this, reliance has to be placed on regular bacteriological analyses to assess potability and to determine the best course of action for protecting the population against waterborne diseases. Drinking water should be clear, cool, free from objectionable tastes and odors and from harmful chemicals and microorganisms of these desired sanitary qualities, 
freedom from harmful microorganisms is most difficult to achieve. It is not impossible, but it demands constant vigilance and repeated testing. This plan was in need of time for design and construction of these plants. So a decision for using the water treatment compact units was taken as a temporary solution till the finish of the big projects. Now and after about 22 years of their application, with the funding from NOPWASD(1985), about 560 of these Compact units have been constructed in Egypt, and the compact units become one of the options for production of potable water as a permanent solution in rural areas of Egypt for both villages and towns (El-in Nadi and Refaat,1995). In the compact units water is treated in the same manner as in conventional systems, but in the compact systems filters are reduced to three sand filters. The methods used for the sanitary water analysis were those recommended by the American Public Health Association (APHA, 1995).In Egypt, the River Nile is the main source of drinking water and other purposes; every effort should be made to achieve drinking water quality as high as practicable, otherwise people life are extremely subjected to hazardous effects. Proper selection and protection of water sources to be used for supplying water treatment systems are of prime importance in the provision of safe drinking water.

Microbial biomass is used to degrade contaminants, nutrients, and organics in wastewater recent developments may mean that biological drinking water treatment may become more feasible and more likely to be accepted by the public. These developments include (1) the rising costs and increasing complexities of handling water-treatment residuals (e.g., membrane concentrates); (2) the emergence of new contaminants that are particularly amenable to biological degradation (e.g., perchlorate); (3) the push for green technologies (i.e., processes that efficiently destroy contaminants instead of concentrating them);(4) regulations limiting the formation of disinfection by-products (DBPs); and (5) the emergence of membrane-based treatment systems, which are highly susceptible to biological fouling ( Abdel-Dayem, 1994). Bacteria gain energy and reproduce by mediating the transfer of electrons from reduced compounds (i.e., compounds that readily donate electrons) to oxidized compounds (i.e., compounds that readily accept electrons). Once electrons are donated by a reduced compound, they travel back and forth across a cell's mitochondrial membrane in a series of internal oxidationreduction reactions. Ultimately, the electrons are donated to the terminal electron-accepting compound. (Madigan et al., 1997).The aim of work is using nano composite( nano polymer) plus bacteria biofilm for treatment surface water in Shebin El-kom city Menofia government - Egypt.

\section{Materials and Methods}

The raw surface water samples of river Nile branch in shebin El-kom city belong to Menofia gov were collected monthly through Mars, July 2012. All water samples were collected according to standards mentioned in (APHA ,1995).Samples were preserved immediately after collection by acidifying with concentrated $\mathrm{HNO}_{3}$ to $\mathrm{pH}<2$ by adding $5 \mathrm{ml}$ nitric acid to 1 liter water samples and in refrigerator.

\section{Bio treatment of raw polluted water}

Non toxic nano polymer composite granules have been prepared by physics dept.; Fac. Sci.

Benha Univ. Three liters of raw surface water were mixed with $60 \mathrm{gm}$ of polymer nano composite (v/w) borne bioagent bacteria as a substrate for biofilm formation. The mixture was aerated for $24 \mathrm{hs}$. These samples of treated water have been uptake for determination the concentration of cations and microbiological analysis.

\section{Physicochemical parameters}

The temperature of treated water samples were measured using a manual thermometer $110\left({ }^{\circ} \mathrm{C}\right)$ graduated to $0.1\left({ }^{\circ} \mathrm{C}\right)$. Turbidity was measured directly by using a digital turbidity meter (WTW). $\mathrm{pH}$ of treated water sample was measured by using a digital $\mathrm{pH}$ meter $(\operatorname{Metrohm}(827 \mathrm{PH}$ lab).

Two cations ( $\mathrm{Fe}$ and $\mathrm{Mn}$ ) were measured in treated water samples using (coupled plasma 400 emission spectrometer Perkin Elemer Emission Spectrometer).Ammonia, Nitrate, Phosphate, Sulfate were measured by the spectrometer (Cecil). Total dissolved solids(TDS) were measured directly by using a digital meter (Conductivity meter selecta).

Conductivity was measured directly by using a digital meter (Conductivity meter selecta).

Free chlorine $0.5 \mathrm{~mL}$ each of buffer reagent and DPD(N,N-diethyl-p-phenyl enediamine) were added to test tube contained $10 \mathrm{~mL}$ of water sample. Mix then read color immediately.

\section{Determination of Coliform groups}

Detection and enumeration of Total Coliform (TC) and Fecal Coliform were determined by Membrane Filter (MF) technique which depends on sample filtration through a 47$\mathrm{mm}, 0.45 \mu \mathrm{m}$ pore size cellulose membrane filters that retains the bacteria present in the sample. The filters were put onto the medium, using a rolling action to avoid trapping air bubbles between the membrane filter and the underlying medium. The plates were inverted and incubated at $35 \pm 0.5\left({ }^{\circ} \mathrm{C}\right)$ for $24 \mathrm{~h}$.

\section{Identification of bacteria isolates}

Biochemical tests using VITEK2 kit for identification of bacteria isolates(Shobra El Khema laboratory of H.C of water and waste water). All the results are within limits of (Egypt Health Ministry,2007), ( WHO , 2007 ) and (Egypt State of Environment Report,2008)

\section{Results}

Under standard and steady-state conditions, the bioreactor was very effective when $20 \mathrm{gm}$ of the nano polymer composite granules were added as a substrate for the biofilm formation for each $1 \mathrm{~L}$ of the raw surface water sample with continuous aeration for $24 \mathrm{~h}$.For the surface water temperature decreased by $2.2^{\circ} \mathrm{C}$ and $2.0^{\circ} \mathrm{C}$ related to raw water and chlorinated water.(TDS) decreased 
from (276 to $220 \mathrm{ppm}$ ) while in chlorinated treatment increase to $(285 \mathrm{ppm})$. $\mathrm{pH}$ increased by 0.2 and $0.4 \mathrm{ppm}$ related to raw water and chlorinated water. Conductivity decrease from(434 to 425) US/CM but increase to(442 $\mathrm{US} / \mathrm{CM}$ ) in the chlorinated treatment while turbidity increase from (9.8 to 10.3) NTU as shown in the table(1) .

The elimination of the heavy metals was remarkable in this study as in rate of Fe that decrease from(0.39 to 0.21$) \mathrm{ppm}$ and for $\mathrm{Mn}$ it remains constant .Total alkalinity decrease from(142 to 130)ppm while in chlorinated treatment was $120 \mathrm{ppm}$. calcium hardness decrease from (78 to 54) ppm and as a result the calcium decrease from (31.2 to 21.6)ppm and in chemical one it has the same value of the raw water sample .Magnesium hardness increase from (100 to 108)ppm \&for the magnesium ion increase from (10.56 to 16.8) ppm. The chlorides decrease to $20 \mathrm{ppm}$ in the bio treated water sample but it was normal to increase to 31 ppm in the chlorinated treated sample. Sulfates content decrease from (15.5 to 10.7)ppm but in chlorinated treatment it increase to $17 \mathrm{ppm}$. Phosphate content increase from $(<0.01$ to 0.02$) \mathrm{ppm}$ while the nitrate content decrease from $(0.86$ to 0.5$) \mathrm{ppm}$ it also decrease in the chlorinated treatment to $0.48 \mathrm{ppm}$ and ammonia content decrease from (0.06 to0.01) ppm and in chlorinated treatment reach to $<0.01 \mathrm{ppm}$ as shown in table (2)

Drinking water sources were contaminated with coliforms and pathogenic bacteria. The bacteriological results shows that all the bio treated samples had no fecal Coliform growth $(<1) \mathrm{C} / 100 \mathrm{ml}$ but give positive results with the total Coliform with confluent colonies whereas in the chlorinated treatment both the total \&fecal Coliform tests were negative as in table (3).

These results indicate that after a moderate exposure to the biofilm as a result to the presence of the nano composite granules, microbial biomass either synthesized or already had the proper enzymes needed for the biosorption of metals so that significant removal of heavy metals was recorded.

Table (1) The physical results of biotreatment \& chlorinated treatment of raw surface water .

\begin{tabular}{|l|l|l|l|l|l|}
\hline Sample & $\begin{array}{l}\text { Cond } \\
\text { US/CM }\end{array}$ & $p H$ & $\begin{array}{l}\text { Turbidity } \\
\text { Before } \\
\text { Filtration } \\
\text { in NTU }\end{array}$ & $\begin{array}{l}\text { T.D.S } \\
p p m\end{array}$ \\
\hline Raw water & 434 & 7.8 & 22.9 & 9.8 & 276 \\
\hline Bio treated water after $24 h$ & 425 & 8.00 & 20.7 & 10.3 & 220 \\
\hline Treated waterwith chloride & 442 & 7.4 & 22 & 0.27 & 285 \\
\hline Max.Value & 1600 & $6.5-8.5$ & ---- & 1 & 1000 \\
\hline
\end{tabular}

\section{Identification of bacteria isolates:-}

Tables(4-17) show the bacterial species were identified according to vitek2 kit tables mainly Gram negative (Acinetobacter haemolyticus, Citrobacter freundii ,Raoultella ornithinolytica, Escherichia coli ,Pseudomonas putida, Aeromonas salmonicida, Klebsiella pneumoniae ssp ozaenae) while (Staphylococcus xylosus, streptococcus agalactiae, Enterococcus casseliflavus, Enterococcus durans, Staphylococcus sciuri, Staphylococcus lentus) were gram positive species.

\section{Discussion}

The present study represented both the physiochemical and bacteriological characteristics of different areas of ground \&surface water in Shebin El-kom city during the period from February till July, 2012 . Temperature is a factor of great importance for aquatic ecosystem, as it affects the microorganism as well as physicochemical properties of water (Delince ,1992). For the surface water temperature decreased by $2.2^{\circ} \mathrm{C}$ and $2.0^{\circ} \mathrm{C}$ related to raw water and chlorinated water as the number of harmful bacteria decrease. (TDS) decreased from (276 to $220 \mathrm{ppm}$ ) while in chlorinated treatment increase to $(285 \mathrm{ppm})$. $\mathrm{pH}$ increased by 0.2 and $0.4 \mathrm{ppm}$ related to raw water and chlorinated water . Conductivity decrease from(434 to 425$)$ US/CM but increase to(442 US/CM) in the chlorinated treatment while turbidity increase from (9.8 to 10.3) NTU. The elimination of the heavy metals was remarkable in this study as in rate of Fe that decrease from (0.39 to 0.21$) \mathrm{ppm}$ and for $\mathrm{Mn}$ it remains constant. Total alkalinity decrease from(142 to 130) ppm while in chlorinated treatment was $120 \mathrm{ppm}$. calcium hardness decrease from (78 to 54) ppm and as a result the calcium decrease from (31.2 to 21.6)ppm and in chemical one it has the same value of the raw water sample. Magnesium hardness increase from (100 to 108 )ppm \&for the magnesium ion increase from (10.56 to 16.8$) \mathrm{ppm}$. The chlorides decrease to $20 \mathrm{ppm}$ in the bio treated watersample but it was normal to increase to $31 \mathrm{ppm}$ in the chlorinated treated sample. Sulfates content decrease from (15.5 to 10.7)ppm but in chlorinated treatment it increase to $17 \mathrm{ppm}$. Phosphate content increase from $(<0.01$ to 0.02$)$ ppm while the nitrate content decrease from $(0.86$ to 0.5$)$ ppm it also decrease in the chlorinated treatment to 0.48 ppm and ammonia content decrease from (0.06 to0.01) ppm and in chlorinated treatment reach to $<0.01 \mathrm{ppm}$. Drinking 
Table (2): The Chemical results of biotreatment \&chlorinated treatment of raw surface water

\begin{tabular}{|c|c|c|c|c|c|c|c|c|c|c|c|c|c|c|}
\hline Sample & $\begin{array}{l}\mathrm{NH3} \\
\mathrm{PPm}\end{array}$ & $\begin{array}{l}\mathrm{NO3} \\
\mathrm{PPm}\end{array}$ & $\begin{array}{l}\text { PO3 } \\
\text { ppm }\end{array}$ & $\begin{array}{l}\mathrm{SO4} \\
\mathrm{ppm}\end{array}$ & $\begin{array}{l}\mathrm{Cl}^{-} \\
\mathrm{ppm}\end{array}$ & $\begin{array}{c}M g+ \\
+ \\
p p m\end{array}$ & $\begin{array}{c}\text { Mg. } \\
\boldsymbol{H} \\
\text { ppm }\end{array}$ & $\begin{array}{l}\mathrm{Ca}++ \\
\mathrm{ppm}\end{array}$ & $\begin{array}{l}\text { Ca.H } \\
\text { ppm }\end{array}$ & $\begin{array}{l}\text { T.H } \\
\text { ppm }\end{array}$ & $\begin{array}{l}\text { T.Alk } \\
\text { ppm }\end{array}$ & $\begin{array}{l}\text { Mn } \\
\text { ppm }\end{array}$ & $\begin{array}{c}F e \\
p p m\end{array}$ & Res.chl \\
\hline Raw water & 0.06 & 0.86 & $<0.01$ & 15.5 & 26 & 10.56 & 44 & 31.2 & 78 & 122 & 142 & $<0.01$ & 0.39 & Nil \\
\hline $\begin{array}{c}\text { Bio treated water } \\
\text { after } 24 \mathrm{~h}\end{array}$ & 0.01 & 0.5 & 0.02 & 10.7 & 20 & 16.8 & 70 & 21.6 & 54 & 124 & 130 & $<0.01$ & 0.21 & Nil \\
\hline $\begin{array}{l}\text { Treated water } \\
\text { with chloride }\end{array}$ & $<0.01$ & 0.48 & $<0.01$ & 17 & 31 & 11.52 & 48 & 31.2 & 78 & 126 & 120 & $<0.01$ & $<0.01$ & 1.4 \\
\hline Max.Value & 0.5 & 45 & Nil & 250 & 250 & ---- & 150 & --- & 350 & 500 & 500 & 0.4 & 0.3 & 1.5 \\
\hline
\end{tabular}

T.H : Total Hardness T.Alk: Total Alkalinity

Ca.H: Calcium Hardness

Table(3): Bacteriological results of biotreatment \&chemical treatment of raw surface water

\begin{tabular}{|c|c|c|c|c|}
\hline Sample & $\begin{array}{c}\text { Total Coliform } \\
\text { C/100ml }\end{array}$ & Final result & $\begin{array}{c}\text { Fecal Coliform } \\
\text { C/100ml }\end{array}$ & Final result \\
\hline Bio treated & Confluent & $+V e$ & $<1$ & $-V e$ \\
\hline Max. Value & $<1$ & $-V e$ & $<1$ & $-V e$ \\
\hline Raw & Confluent & $+V e$ & 2000 & $+V e$ \\
\hline $\begin{array}{c}\text { Treated } \\
\text { with } \text { chloride }\end{array}$ & $<1$ & $-V e$ & $<1$ & $-V e$ \\
\hline
\end{tabular}

Table(4): Selected Organism : Citrobacter Freundii Bionumber :4417610575520011 Confidence: Excellent identification

\begin{tabular}{|c|c|c|c|c|c|c|c|c|c|c|c|c|c|c|c|c|c|}
\hline \multicolumn{18}{|c|}{ Biochemical Details } \\
\hline 2 & APPA & - & 3 & ADO & - & 4 & PyrA & + & 5 & IARL & - & 7 & dCEL & - & 9 & BGAL & + \\
\hline 10 & H25 & + & 11 & BNAG & - & 12 & AGLTp & - & 13 & dGLU & + & 14 & GGT & + & 15 & OFF & + \\
\hline 17 & BGLU & - & 18 & dMAL & + & 19 & dMAN & + & 20 & dMNE & + & 21 & BXYL & - & 22 & BAlap & - \\
\hline 23 & ProA & - & 26 & LIP & - & 27 & PLE & - & 29 & TyrA & + & 31 & URE & - & 32 & dSOR & + \\
\hline 33 & SAC & + & 34 & dTAG & + & 35 & dTRE & + & 36 & CIT & + & 37 & MNT & - & 39 & $5 \mathrm{KG}$ & + \\
\hline 40 & ILATK & + & 41 & AGLU & - & 42 & SUCT & + & 43 & NAGA & - & 44 & AGAL & + & 45 & PHOS & - \\
\hline 46 & GlyA & - & 47 & ODC & - & 48 & LDC & - & 53 & IHISa & - & 56 & CMT & - & 57 & BGUR & - \\
\hline 58 & O129R & + & 59 & GGAA & - & 61 & IMLTa & - & 62 & ELLM & + & 64 & ILATa & - & & & \\
\hline
\end{tabular}


Table(5): Selected Organism : Raoultella ornithinolytica Bionumber : 4627735753773011 Confidence: Good identification

\begin{tabular}{|c|c|c|c|c|c|c|c|c|c|c|c|c|c|c|c|c|c|}
\hline \multicolumn{18}{|c|}{ Biochemical Details } \\
\hline 2 & APPA & - & 3 & ADO & - & 4 & PyrA & + & 5 & IARL & - & 7 & dCEL & + & 9 & BGAL & + \\
\hline 10 & H25 & - & 11 & BNAG & + & 12 & AGLTp & - & 13 & dGLU & + & 14 & GGT & + & 15 & OFF & + \\
\hline 17 & BGLU & + & 18 & dMAL & + & 19 & dMAN & + & 20 & dMNE & + & 21 & BXYL & + & 22 & BAlap & - \\
\hline 23 & ProA & + & 26 & LIP & - & 27 & PLE & + & 29 & TyrA & + & 31 & URE & + & 32 & dSOR & + \\
\hline 33 & SAC & + & 34 & dTAG & - & 35 & dTRE & + & 36 & CIT & + & 37 & MNT & + & 39 & 5KG & - \\
\hline 40 & ILATK & + & 41 & AGLU & + & 42 & SUCT & + & 43 & NAGA & + & 44 & AGAL & + & 45 & PHOS & + \\
\hline 46 & GlyA & + & 47 & ODC & + & 48 & LDC & - & 53 & IHISa & - & 56 & CMT & - & 57 & BGUR & - \\
\hline 58 & O129R & + & 59 & GGAA & - & 61 & IMLTa & - & 62 & ELLM & + & 64 & ILATa & - & & & \\
\hline
\end{tabular}

Table(6): Selected Organism : Escherichia coli Bionumber : 0405610540526611 Confidence: Excellent identification

\begin{tabular}{|c|c|c|c|c|c|c|c|c|c|c|c|c|c|c|c|c|c|}
\hline \multicolumn{18}{|c|}{ Biochemical Details } \\
\hline 2 & APPA & - & 3 & ADO & - & 4 & PyrA & - & 5 & IARL & - & 7 & dCEL & - & 9 & BGAL & + \\
\hline 10 & H25 & - & 11 & BNAG & - & 12 & AGLTp & - & 13 & dGLU & + & 14 & GGT & - & 15 & OFF & + \\
\hline 17 & BGLU & - & 18 & dMAL & + & 19 & dMAN & + & 20 & dMNE & + & 21 & BXYL & - & 22 & BAlap & - \\
\hline 23 & ProA & - & 26 & LIP & - & 27 & PLE & - & 29 & TyrA & + & 31 & URE & - & 32 & dSOR & + \\
\hline 33 & SAC & - & 34 & dTAG & - & 35 & dTRE & + & 36 & CIT & - & 37 & MNT & - & 39 & $5 \mathrm{KG}$ & - \\
\hline 40 & ILATK & + & 41 & AGLU & - & 42 & SUCT & + & 43 & NAGA & - & 44 & AGAL & + & 45 & PHOS & - \\
\hline 46 & GlyA & - & 47 & ODC & + & 48 & LDC & + & 53 & IHISa & - & 56 & CMT & + & 57 & BGUR & + \\
\hline 58 & O129R & + & 59 & GGAA & - & 61 & IMLTa & - & 62 & ELLM & + & 64 & ILATa & - & & & \\
\hline
\end{tabular}

Table(7): Selected Organism : $\quad$ Pseudomonas putida Bionumber : $0003011103500152 \quad$ Confidence: Excellent identification

\begin{tabular}{|c|c|c|c|c|c|c|c|c|c|c|c|c|c|c|c|c|c|}
\hline \multicolumn{18}{|c|}{ Biochemical Details } \\
\hline 2 & APPA & - & 3 & ADO & - & 4 & PyrA & - & 5 & IARL & - & 7 & dCEL & - & 9 & BGAL & - \\
\hline 10 & H25 & - & 11 & BNAG & - & 12 & AGLTp & - & 13 & dGLU & + & 14 & GGT & + & 15 & OFF & - \\
\hline 17 & BGLU & - & 18 & dMAL & - & 19 & dMAN & - & 20 & dMNE & + & 21 & BXYL & - & 22 & BAlap & - \\
\hline 23 & ProA & + & 26 & LIP & - & 27 & PLE & - & 29 & TyrA & + & 31 & URE & - & 32 & dSOR & - \\
\hline 33 & SAC & - & 34 & dTAG & - & 35 & dTRE & - & 36 & CIT & + & 37 & MNT & + & 39 & $5 \mathrm{KG}$ & - \\
\hline 40 & ILATK & + & 41 & AGLU & - & 42 & SUCT & + & 43 & NAGA & - & 44 & AGAL & - & 45 & PHOS & - \\
\hline 46 & GlyA & - & 47 & ODC & - & 48 & LDC & - & 53 & IHISa & + & 56 & CMT & - & 57 & BGUR & - \\
\hline 58 & O129R & + & 59 & GGAA & - & 61 & IMLTa & + & 62 & ELLM & - & 64 & ILATa & + & & & \\
\hline
\end{tabular}


Table (8): Selected Organism : Aeromonas saimonicida

Bionumber : $5010001000001001 \quad$ Confidence: Acceptable identification

\begin{tabular}{|c|c|c|c|c|c|c|c|c|c|c|c|c|c|c|c|c|c|}
\hline \multicolumn{18}{|c|}{ Biochemical Details } \\
\hline 2 & APPA & + & 3 & ADO & - & 4 & PyrA & + & 5 & IARL & - & 7 & dCEL & - & 9 & BGAL & - \\
\hline 10 & H25 & + & 11 & BNAG & - & 12 & AGLTp & - & 13 & dGLU & - & 14 & GGT & - & 15 & OFF & - \\
\hline 17 & BGLU & - & 18 & dMAL & - & 19 & dMAN & - & 20 & dMNE & - & 21 & BXYL & - & 22 & BAlap & - \\
\hline 23 & ProA & + & 26 & LIP & - & 27 & PLE & - & 29 & TyrA & - & 31 & URE & - & 32 & dSOR & - \\
\hline 33 & SAC & - & 34 & dTAG & - & 35 & dTRE & - & 36 & CIT & - & 37 & MNT & - & 39 & 5KG & - \\
\hline 40 & ILATK & - & 41 & AGLU & - & 42 & SUCT & - & 43 & NAGA & - & 44 & AGAL & - & 45 & PHOS & - \\
\hline 46 & GlyA & + & 47 & ODC & - & 48 & LDC & - & 53 & IHISa & - & 56 & CMT & - & 57 & BGUR & - \\
\hline 58 & O129R & - & 59 & GGAA & - & 61 & IMLTa & - & 62 & ELLM & + & 64 & ILATa & - & & & \\
\hline
\end{tabular}

Table (9): Selected Organism : Klebsiella pneumoniae ssp ozaenae

Bionumber : $0401700150260202 \quad$ Confidence: Excellent identification

\begin{tabular}{||c|c|c|c|c|c|c|c|c|c|c|c|c|c|c|c|c|c|}
\hline \multicolumn{10}{|c|}{ Biochemical Details } \\
\hline 2 & APPA & - & 3 & ADO & - & 4 & PyrA & - & 5 & IARL & - & 7 & dCEL & - & 9 & BGAL & + \\
\hline 10 & H25 & - & 11 & BNAG & - & 12 & AGLTp & - & 13 & dGLU & + & 14 & GGT & - & 15 & OFF & - \\
\hline 17 & BGLU & + & 18 & dMAL & + & 19 & dMAN & + & 20 & dMNE & - & 21 & BXYL & - & 22 & BAlap & - \\
\hline 23 & ProA & - & 26 & LIP & - & 27 & PLE & - & 29 & TyrA & + & 31 & URE & - & 32 & dSOR & - \\
\hline 33 & SAC & + & 34 & dTAG & - & 35 & dTRE & + & 36 & CIT & - & 37 & MNT & - & 39 & $5 K G$ & - \\
\hline 40 & ILATK & - & 41 & AGLU & + & 42 & SUCT & - & 43 & NAGA & - & 44 & AGAL & + & 45 & PHOS & + \\
\hline 46 & GlyA & - & 47 & ODC & - & 48 & LDC & - & 53 & IHISa & - & 56 & CMT & + & 57 & BGUR & - \\
\hline 58 & O129R & - & 59 & GGAA & - & 61 & IMLTa & - & 62 & ELLM & - & $\mathbf{6 4}$ & ILATa & + & & \\
\hline
\end{tabular}

Table (10): Selected Organism : Staphylococcus xylosus

Bionumber : $030446010673031 \quad$ Confidence: Excellent identification

Biochemical Details

\begin{tabular}{|c|c|c|c|c|c|c|c|c|c|c|c|c|c|c|c|c|c|}
\hline 2 & AMY & - & 4 & PIPLC & - & 5 & dXYL & - & 8 & ADH1 & + & 9 & BGAL & + & 11 & AGLU & - \\
\hline 13 & APPA & - & 14 & CDEX & - & 15 & AspA & - & 16 & BGAR & - & 17 & AMAN & - & 19 & PHOS & + \\
\hline 20 & LeuA & - & 23 & ProA & - & 24 & BGURr & + & 25 & AGAL & - & 26 & PyrA & + & 27 & BGUR & + \\
\hline 28 & AlaA & - & 29 & TyrA & - & 30 & dSOR & - & 31 & URE & + & 32 & POLYB & - & 37 & dGAL & - \\
\hline 38 & dRIB & - & 39 & ILATK & - & 42 & LAC & - & 44 & NAG & - & 45 & dMAL & + & 46 & BACI & + \\
\hline 47 & NOVO & + & 50 & NC6.5 & + & 52 & dMAN & + & 53 & dMNE & + & 54 & MBdG & + & 56 & PUL & - \\
\hline 57 & dRAF & - & 58 & O129R & - & 59 & SAL & - & 60 & SAC & + & 62 & dTRE & + & 63 & ADH2s & - \\
\hline 64 & OPTO & + & & & & & & & & & & & & & & & \\
\hline
\end{tabular}


Table (11) : Selected Organism : Streptococcus agalactiae

Bionumber :250412223532631

Confidence: Acceptable identification

Biochemical Details

\begin{tabular}{|c|c|c|c|c|c|c|c|c|c|c|c|c|c|c|c|c|c|}
\hline 2 & AMY & - & 4 & PIPLC & + & 5 & dXYL & - & 8 & ADH1 & + & 9 & BGAL & - & 11 & AGLU & + \\
\hline 13 & APPA & - & 14 & CDEX & - & 15 & AspA & - & 16 & BGAR & - & 17 & AMAN & - & 19 & PHOS & + \\
\hline 20 & LeuA & $(+)$ & 23 & ProA & - & 24 & BGURr & - & 25 & AGAL & - & 26 & PyrA & + & 27 & BGUR & - \\
\hline 28 & AlaA & - & 29 & TyrA & + & 30 & dSOR & - & 31 & URE & - & 32 & POLYB & + & 37 & dGAL & - \\
\hline 38 & dRIB & + & 39 & ILATK & + & 42 & LAC & - & 44 & NAG & + & 45 & dMAL & - & 46 & BACI & + \\
\hline 47 & NOVO & + & 50 & NC6.5 & + & 52 & dMAN & - & 53 & dMNE & - & 54 & MBdG & + & 56 & PUL & - \\
\hline 57 & dRAF & - & 58 & O129R & + & 59 & SAL & + & 60 & SAC & + & 62 & dTRE & + & 63 & ADH2s & - \\
\hline 64 & OPTO & + & & & & & & & & & & & & & & & \\
\hline
\end{tabular}

Table (12): Selected Organism : $\quad$ Enterococcus casseliflavus

Bionumber : $160311000341520 \quad$ Confidence: Excellent identification

\begin{tabular}{|c|c|c|c|c|c|c|c|c|c|c|c|c|c|c|c|c|c|}
\hline \multicolumn{18}{|c|}{ Biochemical Details } \\
\hline 2 & AMY & + & 4 & PIPLC & - & 5 & dXYL & - & 8 & ADH1 & - & 9 & BGAL & + & 11 & AGLU & + \\
\hline 13 & APPA & - & 14 & CDEX & - & 15 & AspA & - & 16 & BGAR & + & 17 & AMAN & + & 19 & PHOS & - \\
\hline 20 & LeuA & + & 23 & ProA & - & 24 & BGURr & - & 25 & AGAL & + & 26 & PyrA & - & 27 & BGUR & - \\
\hline 28 & AlaA & - & 29 & TyrA & - & 30 & dSOR & - & 31 & URE & - & 32 & POLYB & - & 37 & dGAL & - \\
\hline 38 & dRIB & - & 39 & ILATK & - & 42 & LAC & - & 44 & NAG & + & 45 & dMAL & + & 46 & BACI & - \\
\hline 47 & NOVO & - & 50 & NC6.5 & - & 52 & dMAN & + & 53 & dMNE & + & 54 & MBdG & - & 56 & PUL & - \\
\hline 57 & dRAF & + & 58 & O129R & - & 59 & SAL & + & 60 & SAC & - & 62 & dTRE & + & 63 & ADH2s & - \\
\hline 64 & OPTO & - & & & & & & & & & & & & & & & \\
\hline
\end{tabular}

Table (13) : Selected Organism : Enterococcus durans

Bionumber : $414002325733661 \quad$ Confidence: Acceptable identification

\begin{tabular}{|c|c|c|c|c|c|c|c|c|c|c|c|c|c|c|c|c|c|}
\hline \multicolumn{18}{|c|}{ Biochemical Details } \\
\hline 2 & AMY & - & 4 & PIPLC & - & 5 & dXYL & + & 8 & ADH1 & + & 9 & BGAL & - & 11 & AGLU & - \\
\hline 13 & APPA & - & 14 & CDEX & - & 15 & AspA & + & 16 & BGAR & - & 17 & AMAN & - & 19 & PHOS & - \\
\hline 20 & LeuA & - & 23 & ProA & - & 24 & BGURr & - & 25 & AGAL & - & 26 & PyrA & + & 27 & BGUR & - \\
\hline 28 & AlaA & + & 29 & TyrA & + & 30 & dSOR & - & 31 & URE & - & 32 & POLYB & + & 37 & dGAL & - \\
\hline 38 & dRIB & + & 39 & ILATK & - & 42 & LAC & + & 44 & NAG & + & 45 & dMAL & + & 46 & BACI & + \\
\hline 47 & NOVO & + & 50 & NC6.5 & + & 52 & dMAN & - & 53 & dMNE & + & 54 & MBdG & + & 56 & PUL & - \\
\hline 57 & dRAF & - & 58 & O129R & + & 59 & SAL & + & 60 & SAC & - & 62 & dTRE & + & 63 & ADH2s & + \\
\hline 64 & OPTO & + & & & & & & & & & & & & & & & \\
\hline
\end{tabular}


Table(14) : Selected Organism : Staphylococcus sciuri

Bionumber : 050002403463431

Confidence: Low discrimination

Biochemical Details

\begin{tabular}{|c|c|c|c|c|c|c|c|c|c|c|c|c|c|c|c|c|c|c|}
\hline 2 & AMY & - & 4 & PIPLC & - & $\mathbf{5}$ & dXYL & - & $\mathbf{8}$ & ADH1 & + & $\mathbf{9}$ & BGAL & $(-)$ & 11 & AGLU & + \\
\hline 13 & APPA & - & $\mathbf{1 4}$ & CDEX & - & $\mathbf{1 5}$ & AspA & - & $\mathbf{1 6}$ & BGAR & - & 17 & AMAN & - & 19 & PHOS & - \\
\hline 20 & LeuA & - & $\mathbf{2 3}$ & ProA & - & $\mathbf{2 4}$ & BGURr & - & $\mathbf{2 5}$ & AGAL & - & $\mathbf{2 6}$ & PyrA & + & 27 & BGUR & - \\
\hline 28 & AlaA & - & $\mathbf{2 9}$ & TyrA & - & $\mathbf{3 0}$ & dSOR & + & $\mathbf{3 1}$ & URE & - & $\mathbf{3 2}$ & POLYB & - & 37 & dGAL & - \\
\hline 38 & dRIB & + & $\mathbf{3 9}$ & ILATK & + & $\mathbf{4 2}$ & LAC & - & $\mathbf{4 4}$ & NAG & - & $\mathbf{4 5}$ & dMAL & $(-)$ & 46 & BACI & + \\
\hline 47 & NOVO & - & $\mathbf{5 0}$ & NC6.5 & + & $\mathbf{5 2}$ & dMAN & + & $\mathbf{5 3}$ & dMNE & + & $\mathbf{5 4}$ & MBdG & + & 56 & PUL & - \\
\hline 57 & dRAF & - & $\mathbf{5 8}$ & O129R & - & $\mathbf{5 9}$ & SAL & + & $\mathbf{6 0}$ & SAC & + & $\mathbf{6 2}$ & dTRE & + & $\mathbf{6 3}$ & ADH2s & - \\
\hline 64 & OPTO & + & & & & & & & & & & & & & & & \\
\hline
\end{tabular}

Table(15): Selected Organism : Staphylococcus lentus

Bionumber : $150003403663731 \quad$ Confidence: Excellent identification

Biochemical Details

\begin{tabular}{|c|c|c|c|c|c|c|c|c|c|c|c|c|c|c|c|c|c|c|}
\hline 2 & AMY & + & 4 & PIPLC & - & 5 & dXYL & - & 8 & ADH1 & + & 9 & BGAL & - & 11 & AGLU & + \\
\hline 13 & APPA & - & 14 & CDEX & - & 15 & AspA & - & 16 & BGAR & - & 17 & AMAN & - & 19 & PHOS & - \\
\hline 20 & LeuA & - & 23 & ProA & - & 24 & BGURr & - & 25 & AGAL & + & 26 & PyrA & + & 27 & BGUR & - \\
\hline 28 & AlaA & - & 29 & TyrA & - & 30 & dSOR & + & 31 & URE & - & 32 & POLYB & - & 37 & dGAL & - \\
\hline 38 & dRIB & + & 39 & ILATK & + & 42 & LAC & - & 44 & NAG & - & 45 & dMAL & + & 46 & BACI & + \\
\hline 47 & NOVO & - & 50 & NC6.5 & + & 52 & dMAN & + & 53 & dMNE & + & 54 & MBdG & + & 56 & PUL & - \\
\hline 57 & dRAF & + & 58 & O129R & + & 59 & SAL & + & 60 & SAC & + & 62 & dTRE & + & 63 & ADH2s & - \\
\hline 64 & OPTO & + & & & & & & & & & & & & & & & \\
\hline
\end{tabular}

Table(16): Selected Organism : Acinetobacter haemolyticus

Bionumber : $0040000101400340 \quad$ Confidence: Excellent identification

\begin{tabular}{|c|c|c|c|c|c|c|c|c|c|c|c|c|c|c|c|c|c|}
\hline \multicolumn{18}{|c|}{ Biochemical Details } \\
\hline 2 & APPA & - & 3 & ADO & - & 4 & PyrA & - & 5 & IARL & - & 7 & dCEL & - & 9 & BGAL & - \\
\hline 10 & H25 & - & 11 & BNAG & - & 12 & AGLTp & + & 13 & dGLU & - & 14 & GGT & - & 15 & OFF & - \\
\hline 17 & BGLU & - & 18 & dMAL & - & 19 & dMAN & - & 20 & dMNE & - & 21 & BXYL & - & 22 & BAlap & - \\
\hline 23 & ProA & - & 26 & LIP & - & 27 & PLE & - & 29 & TyrA & + & 31 & URE & - & 32 & dSOR & - \\
\hline 33 & SAC & - & 34 & dTAG & - & 35 & dTRE & - & 36 & CIT & + & 37 & MNT & - & 39 & 5KG & - \\
\hline 40 & ILATK & - & 41 & AGLU & - & 42 & SUCT & + & 43 & NAGA & - & 44 & AGAL & - & 45 & PHOS & - \\
\hline 46 & GlyA & - & 47 & ODC & - & 48 & LDC & - & 53 & IHISa & + & 56 & CMT & $(+)$ & 57 & BGUR & - \\
\hline 58 & O129R & - & 59 & GGAA & - & 61 & IMLTa & + & 62 & ELLM & - & 64 & ILATa & - & & & \\
\hline
\end{tabular}


Table(17): Selected Organism : Cronobacter dublinensis ssp dublinensis

Bionumber :0625736051723011

Biochemical Details

\begin{tabular}{||c|c|c|c|c|c|c|c|c|c|c|c|c|c|c|c|c|c|c|}
\hline 2 & APPA & - & 3 & ADO & - & 4 & PyrA & - & 5 & IARL & - & 7 & dCEL & + & 9 & BGAL & + \\
\hline 10 & H25 & - & 11 & BNAG & + & 12 & AGLTp & - & 13 & dGLU & + & 14 & GGT & - & 15 & OFF & + \\
\hline 17 & BGLU & + & 18 & dMAL & + & 19 & dMAN & + & 20 & dMNE & + & 21 & BXYL & + & 22 & BAlap & - \\
\hline 23 & ProA & - & 26 & LIP & + & 27 & PLE & + & 29 & TyrA & - & 31 & URE & - & 32 & dSOR & - \\
\hline 33 & SAC & + & 34 & dTAG & - & 35 & dTRE & + & 36 & CIT & + & 37 & MNT & - & 39 & 5KG & - \\
\hline 40 & ILATK & + & 41 & AGLU & + & 42 & SUCT & + & 43 & NAGA & - & 44 & AGAL & + & 45 & PHOS & - \\
\hline 46 & GlyA & + & 47 & ODC & + & 48 & LDC & - & 53 & IHISa & - & 56 & CMT & - & 57 & BGUR & - \\
\hline 58 & O129R & + & 59 & GGAA & - & 61 & IMLTa & - & 62 & ELLM & + & 64 & ILATa & - & & & \\
\hline
\end{tabular}

water sources were contaminated with coliforms and pathogenic bacteria. The bacteriological results shows that all the bio treated samples had no fecal Coliform growth (<1) $\mathrm{C} / 100 \mathrm{ml}$ but give positive results with the total Coliform with confluent colonies whereas in the chlorinated treatment both the total \&fecal Coliform tests were negative.

The use of microbial biomass for the biosorption of metals from industrial and municipal wastewater has been proposed as a promising alternative to conventional heavy metal management strategies in past decades. Fungal, bacterial and yeast biomass have been studied with respect to the adsorption of toxic or strategic metals, mainly due to low production costs, rapid sorption and release of metals and reutilization. Accumulation of metals in microbial biomass proceeds by different processes such as uptake by transport, entrapment in extra cellular capsules, precipitation and oxidation- reduction reactions. Although the mechanism of metal sorption and uptake by microorganisms is still not completely understood. Sorption to poly-saccharides, proteins or other molecules occurring in the outer layer of the cell wall probably plays the most important role. Bioremoval of heavy metal from industrial wastes has been demonstrated by several biotechnologies. Virous microbial species. Gram positive and Gram negative bacteria mainly Pseudomonas (Hussein et al., 2004) and Bacillus (Mayers and Beveridge, 1989), have been shown to relatively efficient in the bioaccumulation of copper, zinc, Iron and other metal ions present in polluted effluents. Generally, the cell walls of Gram negative and Gram positive bacteria consists of an anionic matrix of biopolymers such as peptidoglycan, techoic acid, techuronic acid phospholipid and lipopoly saccharide as well a various poly peptides and poly saccharide. The wall polymers enable bacteria to sorbs and bind significant amounts of metals from their surrounding (Mayers and Beveridge, 1989). Also no generalizations regarding differences between Gram negative and Gram positive for heavy metals sorption metal removal by one or more process (Mullen et al, 1989).

\section{References}

Abdel-Dayem, s. (1994). "Water quality issues in Egypt." Italian-Egyptian Study Days on the Environment, Cairo 9-20 October, pp. 81-92.

American Public Health Association(APHA) (1995).AWWA(American Water Works Assoc.); WPCF (Water Poll. Contr. Fed.). Standard Methods for the Examination of Water and Waste Water. 13th Ed. Washington, DC: Am. Public Health Assoc.; 1995. p. 874.

Delince.(1992). Developments in Hydrobiology; pages: $1-230$.

Egypt State of Environment Report ,2008. Arab republic of Egypt ministry of state for environmental affairs Egyptian environmental affairs agency, September 2008.

Egyptian Health Ministry (2007). Egyptian Standards for Drinking Water.

El-Nadi, and Refaat, A.I. (1995). An overview on the use of compact water treatment units in Egypt. 1st. Int. Conf. on Potable Water Management and Water Treatment Technologies, Cairo, Egypt 5-7 Dec., 1995.

Husein, H.; Ibrahim , S. F.; Kandeel, K. and Moawad, H. (2004). Biosorption of heavy metals from wastewater using Pseudomonas Sp. Electronic J. Biotech. 7 (1): 38-46. 
Madigan, M.T., J.M. Martinko, and J. Parker. 1997. Brock Biology of Microorganisms, 8th ed. Upper Saddle River, N.J.: Prentice Hall.

Mayer,s I.T. and Beveridge, T.J. (1989). The sorption of metals to Bacillu subtilis walls from dilute solution and simulated Hamilton Harbour water. Cond. J. Microbiol. 35(8): 764-770.
Mullen, M.D.; Wolf, D.C.; Ferris , F.G.; Beveridge, T.J.; Flemming, C.A. and Bailey, G.W. (1989). Bacterial sorption of heavy metals. Appl. and Environ. Microbiol . 55(12): 3143-3149.

NOPWASD. National Organization for Potable Water and Sanitary Drainage, (1985).Compact Units Design for Egypt Needs.

World Health Organization, (2007). Guidelines for drinking water quality-I, Recommendations, 2nd Ed. Geneva WHO, 2007. 